\title{
Probiotic intervention in infancy is not associated with development of beta cell autoimmunity and type 1 diabetes
}

\author{
Erkki Savilahti $^{1} \cdot$ Taina Härkönen $^{1,2} \cdot$ Emma M. Savilahti $^{1} \cdot$ Kaarina Kukkonen $^{1} \cdot$ Mikael Kuitunen $^{1} \cdot$ Mikael Knip $^{1,2,3}$
}

Received: 26 June 2018 / Accepted: 24 August 2018 / Published online: 20 September 2018

(C) Springer-Verlag GmbH Germany, part of Springer Nature 2018

Keywords Beta cell autoimmunity · Clinical trial · Probiotics · Type 1 diabetes

To the Editor: The initiators of the autoimmune process in the pancreatic islets leading to type 1 diabetes remain enigmatic. The close association of pancreas with the immunologically highly active gut makes the latter, together with the intestinal microbiota, a prime suspect in the process. Duodenal specimens of individuals with type 1 diabetes have shown distinct inflammation, even in the absence of an HLA genotype compatible with coeliac disease [1]. Furthermore, several inflammatory markers have been shown to be type 1 diabetesspecific in duodenal samples from individuals with type 1 diabetes [2]. Changes in inflammatory markers were associated with alterations in the relative and absolute densities of bacteria in the duodenal specimens [2]. The analysis of microbiota in stool samples from individuals who later progressed to type 1 diabetes suggested that at the time of the appearance of diabetes-associated autoantibodies, the intestinal microbiota shows dysbiosis characterised by decreased bacterial diversity and reduced quantities of bacteria producing butyrate and lactate [3]. Related to the microbiota, exposure to bacterial lipopolysaccharide has been implicated to affect the training of the infant immune system either favouring an increased predisposition to immune-mediated diseases such as type 1 diabetes and allergy or providing relative resistance to such diseases, depending on the specific lipopolysaccharide concerned [4].

The development of autoimmune diabetes in NOD mice was prevented by administration of the probiotic preparation

Erkki Savilahti

erkki.savilahti@helsinki.fi

1 Children's Hospital, University of Helsinki and Helsinki University Hospital, Stenbäckinkatu 11, 00029 HUS, Helsinki, Finland

2 Research Programs Unit, University of Helsinki, Helsinki, Finland

3 Folkhälsan Research Centre, Helsinki, Finland
VSL\#3 (VSL Pharmaceuticals, Ft Lauderdale, FL, USA; containing $3 \times 10^{11} / \mathrm{g}$ viable lyophilised bacteria), comprising a mix of nine different bacteria (Bifidobacterium longum, infantis and breve, Lactobacillus acidophilus, casei, delbrueckii subsp., bulgaricus and plantarum, as well as Streptococcus salivarius subsp. thermophilus) [5]. In the Environmental Determinants of Diabetes in the Young (TEDDY) study, the risk of developing islet cell autoimmunity was reduced among children carrying the high-risk HLA genotype, whose parents reported that they had given probiotics to their baby during the first 4 weeks of life [6]. However, no human randomised trial on the use of probiotics and development of type 1 diabetes or islet cell autoimmunity has been reported.

We performed a randomised, double-blind placebo controlled clinical trial to prevent allergy in a cohort of 1223 children with high risk of allergy, i.e. either or both parents having doctor-diagnosed allergy (the original study has been registered at clinicaltrials.gov, identifier NCT00298337). The ethics committee of the Children's Hospital of University of Helsinki approved the study protocol and mothers of participants gave written informed consent. Mothers of participants were given a capsule of a probiotic preparation containing Lactobacillus GG (ATCC 53103); $5 \times 10^{9}$ colonyforming units [cfu]), Lactobacillus rhamnosus LC705 (DSM $7061 ; 5 \times 10^{9} \mathrm{cfu}$ ), Bifidobacterium breve Bb99 (DSM 13692; $2 \times 10^{8} \mathrm{cfu}$ ), and Propionibacterium freudenreichii ssp. shermanii JS (DSM 7076); $2 \times 10^{9}$ cfu twice a day or a placebo starting from the 36 th gestational week. The infants were given one capsule per day of the same preparation from birth to age 6 months. A detailed clinical follow-up was performed at the ages of 2,5 and 13 years $[7,8]$. The parents were asked at each visit whether their child had been diagnosed with type 1 diabetes.

For the current study, we gathered information on type 1 diabetes diagnoses during the 13 year follow-up. In addition to 
Table 1 Number of upper respiratory tract infections, number of antibiotic treatments and duration of exclusive and total breastfeeding in children developing type 1 diabetes and in the remaining study group

\begin{tabular}{lllllll}
\hline & $\begin{array}{l}\text { Number of } \\
\text { URTI by age } \\
\text { 2 years }\end{array}$ & $\begin{array}{l}\text { Number of URTI } \\
\text { between age 3 and } \\
\text { 6 years }\end{array}$ & $\begin{array}{l}\text { Number of antibiotic } \\
\text { medications by age } \\
\text { 6 months }\end{array}$ & $\begin{array}{l}\text { Number of antibiotic } \\
\text { medications at age 0 } 0 \\
\text { to 2 years }\end{array}$ & $\begin{array}{l}\text { Duration of exclusive } \\
\text { breastfeeding } \\
\text { (months) }\end{array}$ & $\begin{array}{l}\text { Duration of total } \\
\text { breastfeeding } \\
\text { (months) }\end{array}$ \\
\hline $\begin{array}{l}\text { Children with type } 1 \\
\text { diabetes }(n=14)\end{array}$ & $4.0(3.9)$ & $9.3(6.5)$ & $0.14(0.36)$ & $2.1(2.5)$ & $3.0(1.9)$ & $8.3(4.4)$ \\
$\begin{array}{l}\text { Non-diabetic children } \\
\text { (m) }\end{array}$ & $3.4(3.5)^{\mathrm{a}}$ & $7.5(6.1)^{\mathrm{a}}$ & $0.28(0.66)^{\mathrm{a}}$ & $1.6(2.4)^{\mathrm{a}}$ & $2.5(1.9)^{\mathrm{b}}$ & $7.8(5.5)^{\mathrm{c}}$ \\
\hline
\end{tabular}

Data are presented as mean $\pm \mathrm{SD}$

Data available for ${ }^{\mathrm{a}} 1223$ children, ${ }^{\mathrm{b}} 924$ children, ${ }^{\mathrm{c}} 1000$ children

URTI, upper respiratory tract infections

the parents' reporting, the diagnoses of type 1 diabetes were also checked by referring to the Finnish Paediatric Diabetes Registry (coverage $>92 \%$ ) in November 2017, when the youngest participant was 13 years of age. Altogether, 14 children were found to have type 1 diabetes. Among them, seven had received the active probiotic preparation. All those who developed type 1 diabetes had complied with the intervention. Factors known to have an effect on the gut microbiota were similar among those who progressed to diabetes and the remaining participants (Table 1, $p>0.1$ in all comparisons; unpaired $t$ test.) Of those 14 who developed type 1 diabetes, two (14\%) were born by Caesarean section, while the proportion delivered in this way among the remaining children was $17 \%$.

For the current study, in the 649 blood samples obtained at age 5 , we analysed insulin autoantibodies, glutamic acid decarboxylase (aa 96-585), islet antigen 2 and zinc transporter 8 antibodies using previously described methods [9]. Islet cell antibodies were measured only in those who tested positive for any of the four biochemical autoantibodies. We identified 25 children who tested positive for at least one autoantibody (3.9\%), seven of whom (1.1\%) tested positive for multiple $(\geq 2)$ autoantibodies. Among those with at least one autoantibody, $12(48 \%)$ had received the probiotic treatment during infancy. The corresponding proportion among those with multiple autoantibodies was three out of seven (43\%). Daily use of probiotic supplementation (mix of four bacteria) in infancy was thus unrelated to the cumulative incidence of beta cell autoimmunity by the age of 5 years. Use of other, commercially available probiotics as preparations or as additives in foods was reported by parents at follow-up visits and questionnaires (yearly at $0-5$ years, at $5-10$ years and $10-13$ years). Use was not associated with beta cell autoimmunity by 5 years or type 1 diabetes by 13 years of age (data not shown).

In this randomised double-blind placebo-controlled clinical trial designed to study the prevention of allergy, we found no effect of probiotic treatment in infancy on the development of type 1 diabetes by the age of 13 years or on islet cell autoimmunity by the age of 5 years. While the role of the intestinal microbiota appears to be substantial in the development of type 1 diabetes, prospective clinical studies are crucial for a better understanding and for the identification of potential novel preventive strategies.

Data availability The datasets analysed during the current study are not publicly available due to privacy of under-age study subjects but are available from the corresponding author on reasonable request.

Funding This study was funded by the Academy of Finland (grant 292538 and Centre of Excellence in Molecular Systems Immunology and Physiology Research - SyMMyS, Decision No. 250114) and Helsinki University Central Hospital Research Funds.

Duality of interest The authors declare that there is no duality of interest associated with this manuscript.

Contribution statement The corresponding author ES designed the study, interpreted the data and wrote the manuscript. TH was responsible for the analysis of diabetes-associated autoantibodies and participated in the analysis and interpretation of data as well as in the preparation of the manuscript. EMS analysed the data for the study and participated in the interpretation of the data and preparation of the manuscript. KK and M. Kuitunen were responsible for the collection of material for the original probiotic study and its later follow-up, substantially contributed to the acquisition of data and they participated in the preparation of the manuscript. M. Knip substantially contributed to conception and design of the study, in the collection of data for clinical type 1 diabetes and was the head of the laboratory performing the tests for diabetes-associated autoantibodies, and revised the manuscript critically. All authors have approved the final manuscript. Erkki Savilahti is responsible for the integrity of the work as a whole.

\section{References}

1. Westerholm-Ormio M, Garioch J, Ketola I, Savilahti E (2002) Inflammatory cytokines in small intestinal mucosa of patients with potential coeliac disease. Clin Exp Immunol 128:94-101. https://doi. org/10.1046/j.1365-2249.2002.01798.x

2. Pellegrini S, Sordi V, Bolla AM et al (2017) Duodenal mucosa of patients with type 1 diabetes shows distinctive inflammatory profile and microbiota. J Clin Endocrinol Metab 102:1468-1477. https:// doi.org/10.1210/jc.2016-3222

3. Knip M, Siljander H (2016) The role of the intestinal microbiota in type 1 diabetes mellitus. Nat Rev Endocrinol 12:154-167. https:// doi.org/10.1038/nrendo.2015.218

4. Vatanen T, Kostic AD, d'Hennezel E et al (2016) Variation in microbiome LPS immunogenicity contributes to autoimmunity in 
humans. Cell 165:842-853. https://doi.org/10.1016/j.cell.2016.04. 007

5. Calcinaro F, Dionisi S, Marinaro M et al (2005) Oral probiotic administration induces interleukin-10 production and prevents spontaneous autoimmune diabetes in the non-obese diabetic mouse. Diabetologia 48:1565-1575. https://doi.org/10.1007/s00125-005-1831-2

6. Uusitalo U, Liu X, Yang J et al (2016) Association of early exposure of probiotics and islet autoimmunity in the TEDDY study. JAMA Pediatr 170:20-28. https://doi.org/10.1001/jamapediatrics.2015.2757

7. Kukkonen K, Savilahti E, Haahtela T et al (2007) Probiotics and prebiotic galacto-oligosaccharides in the prevention of allergic diseases: a randomized, double-blind, placebocontrolled trial. J Allergy Clin Immunol 119:192-198. https://doi.org/10.1016/j.jaci.2006.09.009

8. Kuitunen M, Kukkonen K, Juntunen-Backman K et al (2009) Probiotics prevent IgE-associated allergy until age 5 years in cesarean-delivered children but not in the total cohort. J Allergy Clin Immunol 123:335-341. https://doi.org/10.1016/j.jaci.2008. 11.019

9. Knip M, Virtanen SM, Seppa K et al (2010) Dietary intervention in infancy and later signs of beta-cell autoimmunity. N Engl J Med 363: 1900-1908. https://doi.org/10.1056/NEJMoa1004809 